\title{
Algebraic Characterization of Observability in Distance-Regular Consensus Networks
}

\author{
Alain Y. Kibangou and Christian Commault
}

\begin{abstract}
In this paper, we study the observability issue in consensus networks modeled with strongly regular graphs or distance regular graphs. We derive a Kalman-like simple algebraic criterion for observability in distance regular graphs. This criterion consists in evaluating the rank of a matrix built with the components of the Bose-Mesner algebra associated with the considered graph. Then, we state a simple necessary condition of observability based on parameters of the graph, namely the diameter, the degree, and the number of vertices of the graph.
\end{abstract}

\section{INTRODUCTION}

During the last years, networked dynamical systems have received great attention; examples include sensor networks, robotic networks, and biological networks to cite few. As a first step, pioneering works were devoted to study agreement protocols, such as average consensus, over networks. A huge amount of algorithms considering various scenarios can be found in the literature (see [1] and references therein for instance). As a second step, analyzing and characterizing properties of networks viewed as dynamical systems arose. One considered issues such as controllability and observability over consensus networks, i.e. a network running a consensus algorithm. Studying the observability of a networked system consists in answering the question: is it possible, for a given node, to reconstruct the entire network state just from its own measurements and those of its neighbors?

Observability plays an important role in distributed estimation and intrusion detection problems [2], [3], [4]. Indeed, in estimating the network state, one can decide if the functioning of the network is normal or not and decide an action to preserve the system functionalities. In consensus networks, observability properties can serve for designing finite-time average consensus protocols such as in [5]. Studying the observability properties of a given network can also help for characterizing the resilience of the network to external attacks such as spying. Therefore, one can constrain the network topology to exhibit some desirable resilience properties. Such a problem is related to the problem of sensor placement and classification in standard systems, [6].

By viewing a consensus network as a linear dynamical system, one can resort to standard matrix theoretic tools for studying observability such as rank test on the Kalman matrix. Such rank test can be cumbersome for large scale networks for instance. By taking the underlying graph structure of network into account, graph theoretic based tools

A. Y. Kibangou is with Gipsa-Lab, University Joseph Fourier, CNRS, Grenoble, France alain.kibangou@ujf-grenoble.fr

C. Commault is with Gipsa-Lab, Grenoble-INP, Grenoble, France christian.commaultegipsa-lab.grenoble-inp. fr have been investigated in the literature, mainly for the dual problem of controllability. Thus, for a single observer case (resp. single leader case for the controllability issue), it has been pointed out that symmetries are obstructions to observability (resp. controllability) [7], [8], [9]. Then, resorting to the notion of equitable partitions of a graph necessary conditions have been stated in [10] for observability over arbitrary graphs and in [7], [8], [11], [12] for controllability. Unfortunately, these conditions are not sufficient except for certain classes of systems (chain and multichain graphs with a single leader at the extremity of the chain [12]). By following a completely different perspective of study, the authors of [13] derive necessary and sufficient conditions for path and ring graphs. Their method, based on rules of number theory, was also applied to simple grid and torus graphs since these graphs can be obtained through Cartesian products of paths and ring graphs respectively [14], [15]. Extension to arbitrary graphs of such approaches seems to be a tough task.

One of the main observations from the literature concerns the difficulty to state graph-theoretic based necessary and sufficient conditions for arbitrary graphs. Therefore, in this paper, our study is focused on families of graphs larger than path or ring graphs, namely strongly regular graphs and distance regular graphs. The regularity properties of these kinds of graphs can particularly be useful for robustifying the network as for cryptographic systems [17]. The main goal of this paper is to re-visit matrix theoretic, or algebraic, tools for characterizing observability in consensus networks. The approach considered herein is based on the Bose-Mesner algebra [18] and the consensus network is not necessarily a Laplacian based one, precisely we consider Max-weights or a Metropolis-Hastings policy.

The paper is organized as follows: in Section II, we first recall some basic properties of graphs and define the class of graphs under study. Then in Section III, we study the observability conditions following an algebraic point of view. The obtained results are illustrated for some particular families of graphs before concluding the paper.

\section{Preliminaries}

At a high level of abstraction, a network can be represented as a graph. We denote by $\mathscr{X}(\mathscr{V} ; \mathscr{E})$ a graph with vertex set $\mathscr{V}=\left\{v_{1}, v_{2}, \cdots, v_{N}\right\}$, of cardinality $|\mathscr{V}|=N$, and edge set $\mathscr{E} \subset \mathscr{V} \times \mathscr{V}$, where an edge is an unordered pair of distinct vertices of $\mathscr{X}$. Usually, the vertex set stands for the set of nodes in the network whereas the edge set allows taking interactions (communications) between nodes into account. 


\section{A. Basic definitions}

For two vertices $v_{i}$ and $v_{j}$ of $\mathscr{X}(\mathscr{V} ; \mathscr{E})$, if $\left(v_{i}, v_{j}\right) \in \mathscr{E}$ then $v_{i}$ and $v_{j}$ are said to be adjacent or neighbors.

Definition 1: A graph $\mathscr{X}(\mathscr{V} ; \mathscr{E})$ with $N$ vertices is said to be:

- empty if it is constituted with $N$ isolated vertices with no edge.

- complete if every pair of distinct vertices is connected by an edge.

- regular of degree (or valency) $K$, when every vertex is precisely adjacent to $K$ vertices.

Since an undirected graph is completely determined by specifying its adjacency structure, it is standard practice to capture such a structure by means of the adjacency matrix $\mathbf{A}$, a binary matrix with entries $\mathbf{A}_{i j}$ defined as: $\mathbf{A}_{i j}=1$ if $\left(v_{i}, v_{j}\right) \in \mathscr{E}$ and zero elsewhere. For regular graphs, we can note that rows and columns of the corresponding adjacency matrix sum exactly to the valency $K$ of the graph:

$$
\mathbf{A} \mathbf{J}_{N}=K \mathbf{J}_{N}, \quad \mathbf{J}_{N} \mathbf{A}=K \mathbf{J}_{N},
$$

where $\mathbf{J}_{N}$ stands for the $N \times N$ all ones matrix.

Now, let us recall some additional basic definitions on graphs:

Definition 2: For a graph $\mathscr{X}(\mathscr{V} ; \mathscr{E})$ :

- A path is a sequence of distinct vertices such that consecutive vertices in the sequence are adjacent. Its length is the number of edges involved.

- A graph is connected if for each pair of distinct vertices there exists a path containing them.

- The distance $\operatorname{dist}\left(v_{i}, v_{j}\right)$ between two vertices $v_{i}$ and $v_{j}$ is the length of the shortest path starting from $v_{i}$ and ending to $v_{j}$.

- The diameter, $D$, of a graph is the maximum distance between any two vertices in $\mathscr{V}$.

After recalling these basic definitions on graphs, now, we define the family of graphs studied in this paper.

B. Distance regular graphs, strongly regular graphs, and the Bose-Mesner algebra

For a graph $\mathscr{X}(\mathscr{V} ; \mathscr{E})$, we denote by $\mathscr{N}_{i}\left(v_{j}\right)$ the $i$ th neighborhood of $v_{j}$; i.e. $\mathscr{N}_{i}\left(v_{j}\right)=\left\{v_{k} \in \mathscr{V}: \operatorname{dist}\left(v_{j}, v_{k}\right)=i\right\}$.

Definition 3: A distance regular graph is a graph $\mathscr{X}(\mathscr{V} ; \mathscr{E})$ with diameter $D$ such that there exists $b_{i}, i=$ $0,1, \cdots, D-1$, and $c_{i}, i=1,2, \cdots, D$, such that for vertices $v_{j}$ and $v_{k}$ with $\operatorname{dist}\left(v_{j}, v_{k}\right)=i$,

$b_{i}=\left|\mathscr{N}_{1}\left(v_{j}\right) \cap \mathscr{N}_{i+1}\left(v_{k}\right)\right| \quad$ and $\quad c_{i}=\left|\mathscr{N}_{1}\left(v_{j}\right) \cap \mathscr{N}_{i-1}\left(v_{k}\right)\right|$.

The array $\left\{b_{0}, \cdots, b_{D-1} ; c_{1}, \cdots, c_{D}\right\}$ is called intersection array of $\mathscr{X}$, with $b_{0} \geq b_{1} \geq \cdots \geq b_{D-1} \geq 0$ and $0<c_{1} \leq$ $c_{2} \leq \cdots \leq c_{D}$.

We can note that $\mathscr{X}$ must be regular with valency $b_{0}=$ $K$ and $c_{1}=1$, since $\mathscr{N}_{0}\left(v_{k}\right)=\left\{v_{k}\right\}$. We can interpret the above definition as follows: given two vertices $v_{j}$ and $v_{k}$ such that $d\left(v_{j}, v_{k}\right)=i$, among the neighbors of $v_{j}$ there are $c_{i}$ at distance $i-1$ from $v_{k}, a_{i}$ at distance $i$, and $b_{i}$ at distance $i+1$, with $a_{i}+b_{i}+c_{i}=K$.
Example 1: A ring graph with $N$ vertices is a distance regular graph with valency 2 . Its intersection array is given by $\{2,1, \cdots, 1 ; 1,1, \cdots, 1,1\}$ if $N$ is odd and $\{2,1, \cdots, 1 ; 1,1, \cdots, 1,2\}$ if $N$ is even.

Definition 4: A graph $\mathscr{X}(\mathscr{V} ; \mathscr{E})$ is said to be strongly regular if it is neither complete nor empty and there are integers $K, a$, and $c$ such that:

1) $\mathscr{X}(\mathscr{V} ; \mathscr{E})$ is regular with valency $K$.

2) Any two adjacent vertices have exactly $a$ common neighbors.

3) Any two distinct non-adjacent vertices have exactly $c$ common neighbors.

A strongly regular graph with $N$ vertices, degree $K$, and parameters $a$ and $c$ is denoted as $\operatorname{SRG}(\mathrm{N}, \mathrm{K}, \mathrm{a}, \mathrm{c})$.

Moreover a connected strongly regular graph $\mathrm{SRG}(\mathrm{N}, \mathrm{K}, \mathrm{a}, \mathrm{c})$ is a distance regular graph of diameter 2 and has intersection array $\{K, K-a-1 ; 1, c\}[16]$.

Let $\mathscr{X}_{i}\left(\mathscr{V} ; \mathscr{E}_{i}\right), i=0, \cdots, D$, be the graph, with adjacency matrices $\mathbf{A}_{i}$, where two vertices are adjacent if their distance in $\mathscr{X}(\mathscr{V} ; \mathscr{E})$ equals $i$. The algebra generated by $\mathscr{A}=\left\{\mathbf{A}_{0}, \cdots, \mathbf{A}_{D}\right\}$ is called the Bose-Mesner algebra of the distance regular graph $\mathscr{X}$ [18]. It fulfills the following properties:

1) $\mathbf{A}_{0}=\mathbf{I}_{N}$, where $\mathbf{I}_{N}$ stands for the $N \times N$ identity matrix,

2) $\sum_{i=0}^{D} \mathbf{A}_{i}=\mathbf{J}_{N}$

3) $\mathbf{A}_{i}^{T} \in \mathscr{A}$ for each $i$,

4) $\mathbf{A}_{i} \mathbf{A}_{j}=\mathbf{A}_{j} \mathbf{A}_{i} \in \operatorname{span}\{\mathscr{A}\}$

5) $\mathbf{A}_{i} \circ \mathbf{A}_{j}=\mathbf{0}$ for $i \neq j$, where $\circ$ denotes the Hadamard product.

A nice property of distance regular graphs that will be useful in our derivations is given as follows, [16]:

$$
\mathbf{A} \mathbf{A}_{i}=b_{i-1} \mathbf{A}_{i-1}+a_{i} \mathbf{A}_{i}+c_{i+1} \mathbf{A}_{i+1} .
$$

We can therefore deduce the following lemma:

Lemma 1: The powers $\mathbf{A}^{m}$ of the adjacency matrix of a distance regular graph with valency $K$, diameter $D$ and intersection array $\left\{b_{0}, \cdots, b_{D-1} ; c_{1}, \cdots, c_{D}\right\}$ can be expanded in the Bose-Mesner algebra $\left\{\mathbf{A}_{0}, \mathbf{A}_{1}, \cdots, \mathbf{A}_{D}\right\}$ as follows:

$$
\mathbf{A}^{m}=\sum_{j=0}^{D} \beta_{m, j} \mathbf{A}_{j}
$$

where the coefficients $\beta_{m, j}$ depend uniquely on the intersection parameters:

$$
\beta_{m, j}=\beta_{m-1, j-1} c_{j}+\beta_{m-1, j} a_{j}+\beta_{m-1, j+1} b_{j},
$$

with $\beta_{m, m}>0, \beta_{m, j}=0$ if $m<j$, and $a_{j}+b_{j}+c_{j}=K$.

Proof: : We can easily verify this property for $m=0$ and $m=1$, we get indeed $\beta_{0,0}=1, \beta_{1,0}=0$, and $\beta_{1,1}=1$. For $m=2$, applying (2), we get $\mathbf{A}^{2}=\mathbf{A} \mathbf{A}_{1}=b_{0} \mathbf{A}_{0}+a_{1} \mathbf{A}_{1}+c_{2} \mathbf{A}_{2}$. Therefore $\beta_{2,0}=b_{0}, \beta_{2,1}=a_{1}, \beta_{2,2}=c_{2}>0$. Now let us assume that the property (3) is fulfilled for $\mathbf{A}^{m-1}$ and let us check the property for $\mathbf{A}^{m}$. Since $\mathbf{A}^{m}=\mathbf{A A}^{m-1}$, we get $\mathbf{A}^{m}=\sum_{j=0}^{D} \beta_{m-1, j} \mathbf{A} \mathbf{A}_{j}$. Applying (2), we can note 
that $\mathbf{A}^{m}=\sum_{j=0}^{D} \beta_{m-1, j}\left(b_{j-1} \mathbf{A}_{j-1}+a_{j} \mathbf{A}_{j}+c_{j+1} \mathbf{A}_{j+1}\right)$. As a consequence, $\mathbf{A}^{m}=\sum_{j=0}^{D} \beta_{m, j} \mathbf{A}_{j}$ with $\beta_{m, j}=\beta_{m-1, j-1} c_{j}+$ $\beta_{m-1, j} a_{j}+\beta_{m-1, j+1} b_{j}$ and $\beta_{m, m}=\beta_{m-1, m-1} c_{m}>0$.

\section{OBSERVABILITY IN CONSENSUS NETWORKS MODELED WITH A DISTANCE REGULAR GRAPH.}

\section{A. Problem formulation}

Let us consider a consensus network with $N$ nodes whose interactions are modeled with a connected regular graph $\mathscr{X}(\mathscr{V} ; \mathscr{E})$ of valency $K$, with adjacency matrix $\mathbf{A}$. The agreement protocol is carried out through a Max-weights policy, which is equivalent to the Metropolis-Hastings one in the case of regular graphs. The dynamics of the network are given by

$$
\mathbf{x}(k+1)=\mathbf{W} \mathbf{x}(k), \quad \mathbf{W}=\frac{1}{K+1}\left(\mathbf{I}_{N}+\mathbf{A}\right),
$$

where $\mathbf{x}(k) \in \mathfrak{R}^{N}$ contains the local values and defines the state of the network.

We assume that each node can directly observe its own value and those of its neighbors. Therefore, for vertex $v_{i}$, the $(K+1) \times N$ observation matrix $\mathbf{C}_{i}$ results from a row selection matrix, i.e. a binary matrix (0-1) having only one nonzero element per row. The observation vector is then given by

$$
\mathbf{y}_{i}(k)=\mathbf{C}_{i} \mathbf{x}(k) .
$$

Our aim is to study the observability of a pair $\left(\mathbf{W}, \mathbf{C}_{i}\right)$ for an arbitrary node $v_{i}$. The focus will be given to connected graphs since non-observability for unconnected graphs is obvious.

Let $\mathscr{C}$ be the set of the $N$ possible observation matrices $\mathbf{C}_{i}, i=1,2, \cdots, N$. In the sequel, we will assume that the first row of $\mathbf{C}_{i}$ allows selecting the state of the vertex of interest, i.e. $v_{i}$. It is well known that observability of the pair (W, $\mathbf{C}$ ), with $\mathbf{C} \in \mathscr{C}$ is guaranteed if and only if the socalled Kalman matrix $\mathbf{O}_{\mathbf{W}, \mathbf{C}}=\left(\begin{array}{c}\mathbf{C} \\ \mathbf{C W} \\ \vdots \\ \mathbf{C W}^{N-1}\end{array}\right) \in \Re^{N(K+1) \times N}$ is full column rank. As observed by [10], we can always check the rank condition to determine the observability of the network. However, it becomes infeasible when the number of nodes becomes very large. Instead we want to be able to guarantee observability when the network is built. That is why it is interesting to investigate how the observability property is related to the topology of the network. Our aim is to show that owing to the Bose-Mesner algebra, observability of distance regular graphs can be characterized by means of simpler matrices.

\section{B. An algebraic observability condition based on the Bose- Mesner algebra}

In order to carry out our study, we will rewrite $\mathbf{O}_{\mathbf{W}, \mathbf{C}}$ according to the matrices $\mathbf{A}_{i}$ defining the Bose-Mesner algebra. First of all, we can note that powers of the state matrix can be written as:

$$
\mathbf{W}^{m}=\sum_{j=0}^{m} \alpha_{m, j} \mathbf{A}^{j}, \quad \text { with } \alpha_{m, j}=\left(\begin{array}{c}
m \\
j
\end{array}\right) \frac{1}{(1+K)^{m}} .
$$

Combining this result with Lemma 3, we can show that rather studying observability through the Kalman matrix, we can instead study a simpler matrix depending on matrices of the Bose-Mesner algebra.

Lemma 2: Consider a network with $N$ nodes modeled with a distance regular graph $\mathscr{X}$ of diameter $D$, valency $K$, and Bose-Mesner algebra generated by $\mathscr{A}=\left\{\mathbf{A}_{0}, \cdots, \mathbf{A}_{D}\right\}$. Assuming that the dynamics of the network are modeled with equations (4) and (5), the pair $(\mathbf{W}, \mathbf{C}), \mathbf{C} \in \mathscr{C}$, is observable if and only if the matrix $\hat{\mathbf{O}}_{\mathbf{C}}=\left(\begin{array}{c}\mathbf{C A}_{0} \\ \mathbf{C A}_{1} \\ \vdots \\ \mathbf{C A}_{D}\end{array}\right) \in \Re^{(D+1)(K+1) \times N}$ is full column rank.

Proof: : From (6), let $\boldsymbol{\Phi}$ be the $N \times N$ nonsingular triangular matrix of entries $\alpha_{m, j}$. We get:

$$
\left(\begin{array}{c}
\mathbf{W}^{0} \\
\mathbf{W} \\
\vdots \\
\mathbf{W}^{N-1}
\end{array}\right)=\left(\boldsymbol{\Phi} \otimes \mathbf{I}_{N}\right)\left(\begin{array}{c}
\mathbf{A}^{0} \\
\mathbf{A} \\
\vdots \\
\mathbf{A}^{N-1}
\end{array}\right)
$$

where $\otimes$ stands for the Kronecker matrix product. Now, using Lemma 1, we get:

$$
\left(\begin{array}{c}
\mathbf{A}^{0} \\
\mathbf{A} \\
\vdots \\
\mathbf{A}^{N-1}
\end{array}\right)=\left(\boldsymbol{\Psi} \otimes \mathbf{I}_{N}\right)\left(\begin{array}{c}
\mathbf{A}_{0} \\
\mathbf{A}_{1} \\
\vdots \\
\mathbf{A}_{D}
\end{array}\right),
$$

where $\boldsymbol{\Psi}$ denotes the $N \times(D+1)$ lower trapezoidal matrix with $\beta_{m, j}$ as entries. Since the diagonal entries $\beta_{m, m}$ of $\Psi$ are strictly positive and since $N \geq D+1, \Psi$ is full column rank, i.e. $\operatorname{rank}(\Psi)=D+1$.

Now, the observability matrix $\mathbf{O}_{\mathbf{W}, \mathbf{C}_{i}}$ can be rewritten as

$$
\mathbf{O}_{\mathbf{W}, \mathbf{C}}=\left(\mathbf{I}_{N} \otimes \mathbf{C}\right)\left(\boldsymbol{\Phi} \otimes \mathbf{I}_{N}\right)\left(\boldsymbol{\Psi} \otimes \mathbf{I}_{N}\right)\left(\begin{array}{c}
\mathbf{A}_{0} \\
\vdots \\
\mathbf{A}_{D}
\end{array}\right) .
$$

Defining $\boldsymbol{\Gamma}=\boldsymbol{\Phi} \Psi \in \mathfrak{R}^{N \times(D+1)}$, then using properties of the Kronecker product we can note that

$$
\begin{aligned}
\left(\mathbf{I}_{N} \otimes \mathbf{C}\right)\left(\boldsymbol{\Phi} \otimes \mathbf{I}_{N}\right)\left(\boldsymbol{\Psi} \otimes \mathbf{I}_{N}\right) & =\left(\mathbf{I}_{N} \otimes \mathbf{C}\right)\left(\boldsymbol{\Gamma} \otimes \mathbf{I}_{N}\right) \\
& =\left(\boldsymbol{\Gamma} \otimes \mathbf{I}_{K+1}\right)\left(\mathbf{I}_{D+1} \otimes \mathbf{C}\right) .
\end{aligned}
$$

Therefore

$$
\mathbf{O}_{\mathbf{W}, \mathbf{C}}=\left(\boldsymbol{\Gamma} \otimes \mathbf{I}_{K+1}\right)\left(\begin{array}{c}
\mathbf{C A}_{0} \\
\vdots \\
\mathbf{C A}_{D}
\end{array}\right)=\left(\boldsymbol{\Gamma} \otimes \mathbf{I}_{K+1}\right) \hat{\mathbf{O}}_{\mathbf{C}} .
$$

$\boldsymbol{\Phi}$ being a nonsingular matrix, $\operatorname{rank}(\boldsymbol{\Gamma})=\operatorname{rank}(\boldsymbol{\Psi})=D+$ 1. Moreover, from properties of the rank of a Kronecker 
product, $\operatorname{rank}\left(\boldsymbol{\Gamma} \otimes \mathbf{I}_{K+1}\right)=(D+1)(K+1)$, meaning that $\boldsymbol{\Gamma} \otimes \mathbf{I}_{K+1} \in \mathfrak{R}^{N(K+1) \times(D+1)(K+1)}$ is full column rank. Finally, we conclude that $\operatorname{rank}\left(\mathbf{O}_{\mathbf{W}, \mathbf{C}}\right)=\operatorname{rank}\left(\hat{\mathbf{O}}_{\mathbf{C}}\right)$. Hence, the pair $(\mathbf{W}, \mathbf{C})$ is observable iff $\hat{\mathbf{O}}_{\mathbf{C}}$ is full column rank.

The matrix $\hat{\mathbf{O}}_{\mathbf{C}}$ is simpler than the Kalman matrix. Indeed, it is smaller and it does not resort to powers of the network matrix. The matrices $\mathbf{C A}_{l}$ involved in $\hat{\mathbf{O}}_{\mathbf{C}}$ have also some nice properties. For instance the following result will be particularly useful in the sequel.

Lemma 3: The first row $\mathbf{w}_{1, l}^{T}$ of the matrix $\mathbf{C A}_{l}, \mathbf{C} \in \mathscr{C}$ can be written as a linear combination of rows of $\mathbf{C A}_{j}, j=$ $0,1, \cdots, l-1$.

Proof: : Let $\mathbf{e}_{j}$ be the $j$-th vector of the canonical basis of $\mathfrak{R}^{N}$. For an arbitrary matrix $\mathbf{M}$, the scalar $\mathbf{1}^{T} \mathbf{M e}$, where 1 stands for an all ones column-vector with compatible dimension, results from the sum of the entries of the $j$-th column of $\mathbf{M}$. Let $\mathbf{C}$ be the observation matrix associated with an arbitrary vertex $v_{k}$. From properties of distance regular graphs, we know that if $\operatorname{dist}\left(v_{k}, v_{j}\right)=i$ then $c_{i}$ neighbors of vertex $v_{k}$ are at distance $i-1$ of $j$, i.e. $\mathbf{1}^{T} \mathbf{C A}_{i-1} \mathbf{e}_{j}=c_{i}$. In addition, we know that $b_{i}$ (resp. $a_{i}$ ) neighbors of $v_{k}$ are at distance $i+1$ (resp. $i$ ) of $v_{j}$. Therefore, $\mathbf{1}^{T} \mathbf{C A}_{i+1} \mathbf{e}_{j}=b_{i}$ and $\mathbf{1}^{T} \mathbf{C} \mathbf{A}_{i} \mathbf{e}_{j}=a_{i}+1$ since $\operatorname{dist}\left(v_{k}, v_{j}\right)=i$. We also note that the entries of $\mathbf{w}_{1, l}^{T}$ can be written as $\mathbf{w}_{1, l}^{T} \mathbf{e}_{j}$, $j=1, \cdots, N$. They are equal to zero or one, with $\mathbf{w}_{1, l}^{T} \mathbf{e}_{j}=1$ meaning that $\operatorname{dist}\left(v_{k}, v_{j}\right)=l$. Since from parameters of the intersection array we get aggregate informations on rows of $\mathbf{C A}_{j}$, we assume that $\mathbf{w}_{1, l}^{T}$ can be written as $\mathbf{w}_{1, l}^{T}=$ $\mu_{0, l} \mathbf{w}_{1,0}^{T}+\sum_{m=0}^{l-1} \mu_{m+1, l} \mathbf{1}^{T} \mathbf{C} \mathbf{A}_{m}$. Several cases are to be taken into account:

For $j \neq k$, let $\mathbf{w}_{1, l}^{T} \mathbf{e}_{j}$ be a nonzero entry of $\mathbf{w}_{1, l}^{T}$, meaning that $\operatorname{dist}\left(v_{k}, v_{j}\right)=l$. We get:

$$
1=\mathbf{w}_{1, l}^{T} \mathbf{e}_{j}=\sum_{m=0}^{l-1} \mu_{m+1, l} \mathbf{1}^{T} \mathbf{C} \mathbf{A}_{m} \mathbf{e}_{j} .
$$

Since $\operatorname{dist}\left(v_{k}, v_{j}\right)=l$, only $\mathbf{1}^{T} \mathbf{C} \mathbf{A}_{m} \mathbf{e}_{j}, m=l-1, l, l+1$, are nonzero. Hence:

$$
1=\mathbf{w}_{1, l}^{T} \mathbf{e}_{j}=\mu_{l, l} \mathbf{1}^{T} \mathbf{C} \mathbf{A}_{l-1} \mathbf{e}_{j}=\mu_{l, l} c_{l} .
$$

Now, for a zero entry of $\mathbf{w}_{1, l}^{T}$, i.e. $\mathbf{w}_{1, l}^{T} \mathbf{e}_{j}=0$, we know that $\operatorname{dist}\left(v_{k}, v_{j}\right) \neq l$. As a consequence, there exists $q \neq l$ such that $\operatorname{dist}\left(v_{k}, v_{j}\right)=q$, meaning that $\mathbf{w}_{1, q}^{T} \mathbf{e}_{j}=1$. As a consequence, we get $\mathbf{1}^{T} \mathbf{C} \mathbf{A}_{q-1} \mathbf{e}_{j}=c_{q}, \mathbf{1}^{T} \mathbf{C} \mathbf{A}_{q} \mathbf{e}_{j}=a_{q}+1$, and $\mathbf{1}^{T} \mathbf{C A}_{q+1} \mathbf{e}_{j}=b_{q}$. For $q>l$, the involved sum is zero. For $q<l-1$, we get:

$$
\begin{aligned}
0= & \mathbf{w}_{1, l}^{T} \mathbf{e}_{j}=\mu_{q, l} \mathbf{1}^{T} \mathbf{C A}_{q-1} \mathbf{e}_{j}+\mu_{q+1, l} \mathbf{1}^{T} \mathbf{C A}_{q} \mathbf{e}_{j} \\
& +\mu_{q+2, l} \mathbf{1}^{T} \mathbf{C A}_{q+1} \mathbf{e}_{j} \\
= & \mu_{q, l} c_{q}+\mu_{q+1, l}\left(a_{q}+1\right)+\mu_{q+2, l} b_{q}
\end{aligned}
$$

while for $q=l-1$ :

$$
\begin{aligned}
0 & =\mathbf{w}_{1, l}^{T} \mathbf{e}_{j}=\mu_{l-1, l} \mathbf{1}^{T} \mathbf{C} \mathbf{A}_{l-2} \mathbf{e}_{j}+\mu_{l, l} \mathbf{1}^{T} \mathbf{C} \mathbf{A}_{l-1} \mathbf{e}_{j} \\
& =\mu_{l-1, l} c_{l-1}+\mu_{l, l}\left(a_{l-1}+1\right)
\end{aligned}
$$

For $j=k$, we get $0=\mathbf{w}_{1, l}^{T} \mathbf{e}_{k}=\mu_{0, l}+\mu_{1, l}\left(a_{0}+1\right)+b_{0} \mu_{2, l}$. Since $v_{k}$ has at least one vertex $v_{j}$ at distance $m, m=$
$0,1, \cdots, l$, then, combining the $l+1$ independent equations obtained above we get: $\mathbf{P} \boldsymbol{\mu}_{l}=\left(\begin{array}{llll}0 & 0 & \ldots & 1\end{array}\right)^{T}$ with

$$
\mathbf{P}=\left(\begin{array}{ccccccc}
1 & a_{0}+1 & b_{0} & 0 & \cdots & \cdots & 0 \\
0 & c_{1} & a_{1}+1 & b_{1} & 0 & \cdots & 0 \\
0 & 0 & c_{2} & a_{2}+1 & b_{2} & & \vdots \\
0 & 0 & 0 & c_{3} & \ddots & \ddots & 0 \\
\vdots & \vdots & \vdots & & \ddots & \ddots & b_{l-2} \\
& & & & & \ddots & a_{l-1}+1 \\
0 & 0 & \ldots & \ldots & \ldots & 0 & c_{l}
\end{array}\right)
$$

and $\boldsymbol{\mu}_{l}^{T}=\left(\begin{array}{llll}\mu_{0, l} & \mu_{1, l} & \ldots & \mu_{l, l}\end{array}\right)$. Since $c_{i}>0, \quad i=$ $1, \cdots, D$, the system of equations above admits a unique solution. Therefore for any $l, \mathbf{w}_{1, l}^{T}$ can be written as a linear combination of the rows associated with $\mathbf{C}_{k} \mathbf{A}_{j}, j=$ $0,1, \cdots, l-1$.

Example 2: To illustrate the result of Lemma 3, let us consider the case of a ring graph with 6 nodes. The corresponding equivalent observability matrix $\hat{\mathbf{O}}_{1}$, associated with the vertex $v_{1}$, is given by:

$$
\hat{\mathbf{O}}_{1}=\left(\begin{array}{cccccc}
1 & 0 & 0 & 0 & 0 & 0 \\
0 & 1 & 0 & 0 & 0 & 0 \\
0 & 0 & 0 & 0 & 0 & 1 \\
\hline 0 & 1 & 0 & 0 & 0 & 1 \\
1 & 0 & 1 & 0 & 0 & 0 \\
1 & 0 & 0 & 0 & 1 & 0 \\
\hline 0 & 0 & 1 & 0 & 1 & 0 \\
0 & 0 & 0 & 1 & 0 & 1 \\
0 & 1 & 0 & 1 & 0 & 0 \\
\hline 0 & 0 & 0 & 1 & 0 & 0 \\
0 & 0 & 0 & 0 & 1 & 0 \\
0 & 0 & 1 & 0 & 0 & 0
\end{array}\right)
$$

The intersection array associated with this graph is $\{2,1,1 ; 1,1,2\}$. According to Lemma 3, by solving

$$
\left(\begin{array}{llll}
1 & 1 & 2 & 0 \\
0 & 1 & 1 & 1 \\
0 & 0 & 1 & 1 \\
0 & 0 & 0 & 2
\end{array}\right)\left(\begin{array}{l}
\mu_{0,3} \\
\mu_{1,3} \\
\mu_{2,3} \\
\mu_{3,3}
\end{array}\right)=\left(\begin{array}{l}
0 \\
0 \\
0 \\
1
\end{array}\right)
$$

we get the coefficients $\mu_{0,3}=1, \mu_{1,3}=0, \mu_{2,3}=-1 / 2$, and $\mu_{3,3}=1 / 2$. Therefore the 10 th row of $\hat{\mathbf{O}}_{1}$, denoted $\mathbf{w}_{1,3}^{T}$ can be written as:

$$
\begin{aligned}
\mathbf{w}_{1,3}^{T}= & \mu_{0,3} \mathbf{w}_{1,0}^{T}+\mu_{2,3}\left(\mathbf{w}_{1,1}^{T}+\mathbf{w}_{2,1}^{T}+\mathbf{w}_{3,1}^{T}\right) \\
& +\mu_{3,3}\left(\mathbf{w}_{1,2}^{T}+\mathbf{w}_{2,2}^{T}+\mathbf{w}_{3,2}^{T}\right) .
\end{aligned}
$$

Now, equivalently to the Kalman matrix, we define a BoseMesner observability matrix that completely characterizes observability in distance regular graphs.

Theorem 1: Consider a network with $N$ nodes modeled with a distance regular graph $\mathscr{X}$ of diameter $D$ and valency $K$, whose Bose-Mesner algebra is given by $\mathscr{A}=$ $\left\{\mathbf{A}_{0}, \cdots, \mathbf{A}_{D}\right\}$. Let $\mathbf{C} \in \mathscr{C}$ be an observation matrix consistent 
with the dynamics of the network modeled with equations (4) and (5). The pair $(\mathbf{W}, \mathbf{C})$ is observable if and only if the Bose-Mesner observability matrix $\overline{\mathbf{O}}=\left(\begin{array}{c}\mathbf{C A}_{0} \\ \vdots \\ \mathbf{C A}_{D-1}\end{array}\right) \epsilon$ $\mathfrak{R}^{D(K+1) \times N}$ is full column rank.

Proof: : From properties of the Bose-Mesner algebra we know that $\sum_{j=0}^{D} \mathbf{A}_{j}=\mathbf{J}_{N}$, which yields $\sum_{j=0}^{D} \mathbf{C} \mathbf{A}_{j}=\mathbf{J}_{(K+1) \times N}$, where $\mathbf{J}_{(K+1) \times N}=\mathbf{C} \mathbf{J}_{N}$ stands for a $(K+1) \times N$ all ones matrix. We can conclude that a row of $\mathbf{C A}_{D}$ can be written as a linear combination of the all ones row vector $\mathbf{1}^{T}$ and those of matrices $\mathbf{C A}_{j}, j=0,1, \cdots, D-1$. More precisely if $\mathbf{w}_{j, l}^{T}$ denotes the $j$-th row of the matrix $\mathbf{C A}_{l}$, then we have $\mathbf{w}_{j, D}^{T}=\mathbf{1}^{T}-\sum_{l=0}^{D-1} \mathbf{w}_{j, l}^{T}$. We can then conclude that $\mathbf{w}_{j, D}^{T}=$ $\sum_{l=0}^{D} \mathbf{w}_{1, l}^{T}-\sum_{l=0}^{D-1} \mathbf{w}_{j, l}^{T}, j=2, \cdots, K+1$. As a consequence the $K$ last rows of $\mathbf{C A}_{D}$ do not increase the rank of $\hat{\mathbf{O}}$. Thus $\operatorname{rank}(\hat{\mathbf{O}})=\operatorname{rank}\left(\left(\begin{array}{c}\mathbf{C A}_{0} \\ \vdots \\ \mathbf{C A}_{D-1} \\ \mathbf{w}_{1, D}^{T}\end{array}\right)\right)$. Now, using Lemma 3, we know that $\mathbf{w}_{1, D}^{T}$ is a linear combination of rows of $\mathbf{C A} \mathbf{A}_{j}$, $j=0,1, \cdots, D-1$. We can therefore state that $\operatorname{rank}(\hat{\mathbf{O}})=$ $\operatorname{rank}\left(\left(\begin{array}{c}\mathbf{C A}_{0} \\ \vdots \\ \mathbf{C A}_{D-1}\end{array}\right)\right.$, which concludes the proof.

The Bose-Mesner observability matrix is much simpler than the Kalman matrix. Given, the adjacency matrix the computation of $\mathbf{A}_{l}$ has the same complexity than that of $\mathbf{A}^{l}$. Since in general the diameter $D$ is much lower than $N$, the computational cost of the Bose-Mesner observability matrix is then much lower than that of Kalman matrix. Furthermore, we can also make use of the result of Lemma 3 for excluding the first rows of $\mathbf{C A}_{d}, d=1,2, \cdots, D-1$, in the evaluation of the rank of the Bose-Mesner observability matrix. Indeed, let $\overline{\mathbf{C}}$ be the matrix obtained by excluding the first row of $\mathbf{C} \in \mathscr{C}$. The matrices $\tilde{\mathbf{O}}=\left(\begin{array}{c}\mathbf{C A}_{0} \\ \overline{\mathbf{C}} \mathbf{A}_{1} \\ \vdots \\ \overline{\mathbf{C}} \mathbf{A}_{D-1}\end{array}\right) \in \mathfrak{R}^{(D K+1) \times N}$ and the Bose-Mesner observability matrix have the same rank. We define a reduced Bose-Mesner observability matrix as follows:

$$
\overline{\mathbf{0}}=\left(\begin{array}{c}
\overline{\mathbf{C}} \mathbf{A}_{1} \mathbf{S} \\
\vdots \\
\overline{\mathbf{C}} \mathbf{A}_{D-1} \mathbf{S}
\end{array}\right) \in \Re^{(D-1) K \times(N-K-1)}
$$

where $\mathbf{S}$ denotes a column selection matrix such that $\overline{\mathbf{C}} \mathbf{S}=$ $\mathbf{0}_{K \times\left(N_{K}-1\right)}$. We can therefore state the following theorem:

Theorem 2: Consider a network with $N$ nodes modeled with a distance regular graph $\mathscr{X}$ of diameter $D$ and valency $K$ whose Bose-Mesner algebra is given by $\mathscr{A}=$ $\left\{\mathbf{A}_{0}, \cdots, \mathbf{A}_{D}\right\}$. The pair $(\mathbf{W}, \mathbf{C})$ is observable if and only if the reduced Bose-Mesner observability matrix (7) is full column rank.

Proof: As said previously observability is ensured if and only if $\tilde{\mathbf{O}}$ is full column rank. Let $\boldsymbol{\Pi}$ be a permutation matrix so that

$$
\tilde{\mathbf{O}} \boldsymbol{\Pi}=\left(\begin{array}{cc}
\mathbf{I}_{K+1} & \mathbf{0}_{(K+1) \times(N-K-1)} \\
\mathbf{Z} & \tilde{\mathbf{O}}
\end{array}\right) .
$$

We can easily show that $\operatorname{rank}(\tilde{\mathbf{O}})=\operatorname{rank}(\tilde{\mathbf{O}} \boldsymbol{\Pi})=K+1+$ $\operatorname{rank}(\overline{\mathbf{\mathbf { O }}}),[19]$. This rank equals $N$ if and only if the reduced Bose-Mesner observability matrix has rank $N-K-1$.

As a consequence we get the following necessary condition similar to that obtained for controllability in Laplacian based consensus in multi-agents systems [11].

Corollary 1: Consider a network with $N$ nodes modeled with a distance regular graph $\mathscr{X}$ of diameter $D$ and valency $K$ whose Bose-Mesner algebra is given by $\mathscr{A}=$ $\left\{\mathbf{A}_{0}, \cdots, \mathbf{A}_{D}\right\}$. Let $\mathbf{C} \in \mathscr{C}$ be an observation matrix consistent with the dynamics of the network modeled with equations (4) and (5). The pair $(\mathbf{W}, \mathbf{C})$ is observable only if $D K \geq N-1$.

This result allows stating on non-observability of any pair $(\mathbf{W}, \mathbf{C})$ just by considering the diameter, the valency, and the number of nodes of the graph.

Example 3: The Petersen graph (see Fig. 1) is a well known strongly regular graphs with parameters SRG(10,3,0,1). Using Corollary 1, we can directly con-

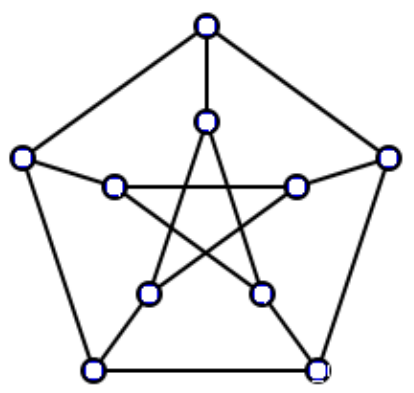

Fig. 1. Petersen graph.

clude that any pair $(\mathbf{W}, \mathbf{C})$ is not observable when the underlying network topology is modeled with a Petersen graph. Indeed the valency is lower than the required lower bound. Similarly, we can deduce that nonobservability is faced by the following strongly regular graphs (see [20] and [16] for complete description of these graphs): Brouwer-Haemers (SRG $(81,20,1,6)$ ), HigmanSims (SRG(100,22,0,6)), M22 (SRG(77,16,0,4)), HoffmanSingleton (SRG(50,7,0,1)), Sims-Gewirtz (SRG $(56,10,0,2))$. One can note that the condition stated in Corollary 1 is just necessary as illustrated by the following example:

Example 4: The Hamming graph $\mathrm{H}(\mathrm{d}, \mathrm{q})$ has vertex set the set of ordered $d$-tuples of elements of $\mathscr{S}$, or sequences of length $d$ from $\mathscr{S}$, where $\mathscr{S}$ stands for a set of $q$ elements. Two vertices are adjacent if they differ in precisely one coordinate. A Hamming graph $\mathrm{H}(\mathrm{d}, \mathrm{q})$ can also be viewed as the result of a cartesian product of $d$ complete graphs with $q$ vertices. It is distance regular with a number of vertices 
$N=q^{d}$, diameter $d$, and degree $d(q-1)$. According to the necessary condition derived in Corollary 1 , we know that observability is ensured only if $d(q-1) \geq \frac{q^{d}-1}{d}$; meaning that the bi-variate function $f(d, q)=q^{d}-d^{2}(q-1)-1$ should be non positive. For $d=4$ and $q=2, f(d, q)$ is non positive, meaning that the necessary condition is fulfilled. According to Theorem 2, the corresponding reduced BoseMesner observability matrix should be a rank 11 matrix. However, actually the rank of this matrix is equal to 9. As a consequence, $\mathrm{H}(4,2)$ is not observable even though it fulfills the condition in Corollary 1.

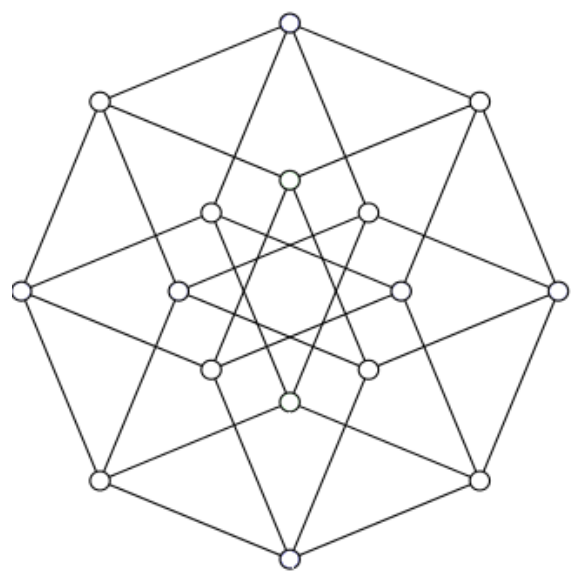

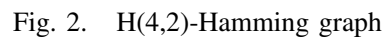

Example 5: A $n \times n$ Rook's graph is a SRG with parameters $\left(n^{2}, 2 n-2, n-2,2\right), n \geq 2$. It represents the moves of a rook on an $n \times n$ chessboard. Its vertices may be given coordinates $\left(x_{1}, x_{2}\right)$, where $1 \leq x_{i} \leq n, i=1,2$. Two vertices are adjacent if and only if they have one common coordinate. They can be viewed as $\mathrm{H}(2, \mathrm{n})$-Hamming graphs. From Corollary 1, we can deduce that a $n \times n$ Rook's graph is observable only for the values of $n$ giving rise to non positive values for the polynomial $f(n)=n^{2}-4 n+3$. Only two values of $n$ fulfill this condition: $n=2$ and $n=3$. For $n=2$ the reduced Bose-Mesner observability matrix is a nonzero vector. Observability is then guaranteed. However for $n=3$, the reduced Bose-Mesner observability matrix

$$
\overline{\tilde{\mathbf{O}}}=\left(\begin{array}{llll}
1 & 1 & 0 & 0 \\
1 & 0 & 1 & 0 \\
0 & 1 & 0 & 1 \\
0 & 0 & 1 & 1
\end{array}\right)
$$

is rank deficient that implies non-observability. Note that the Rook's graph with $n=2$ is a cycle graph with 4 vertices.

\section{CONCLUSION}

In this paper, we have studied the observability issue in a consensus network described with a connected undirected graph with a topology constrained to be strongly regular or distance regular. We have derived an algebraic condition for observability based on the Bose-Mesner algebra. In particular, we have introduced two new matrices for characterizing observability in such graphs. From these matrices, we have deduced a necessary condition: observability is ensured in such graphs only if $D K \geq N-1$ where $D$ is diameter of the graph, $N$ the number of vertices, and $K$ the valency of the graph, i.e. the cardinality of the neighborhood. Instead of a real matrix, the rank test in the proposed method concerns binary matrices. Therefore, in our current works, We are investigating a graph characterization of observability based on the structure of the reduced Bose-Mesner observability matrix.

\section{REFERENCES}

[1] F. Garin and L. Schenato. A survey on distributed estimation and control applications using linear consensus algorithms. In Alberto Bemporad, Maurice Heemels, and Mikael Johansson, editors, Networked Control Systems, volume 406 of Lecture Notes in Control and Information Sciences, pages 75-107. Springer, 2011.

[2] S. Sundaram and C.N. Hadjicostis. Distributed function calculation via linear iterative strategies in the presence of malicious agents. IEEE Trans. on Automatic Control, 56(7):1495-1508, July 2011.

[3] F. Pasqualetti, A. Bicchi, and F. Bullo. Consensus computation in unreliable networks: A system theoretic approach. IEEE Trans. on Automatic Control, 57(1):90-104, 2012.

[4] A.Y. Kibangou and C. Commault. Decentralized Laplacian eigenvalues estimation and collaborative network topology identification. In $3 \mathrm{rd}$ IFAC Workshop on Distributed Estimation and Control in Networked Systems (NecSys'12), Santa Barbara, United States, September 2012.

[5] S. Sundaram and C. Hadjicostis. Distributed function calculation and consensus using linear iterative strategies. IEEE Journal on Selected Areas in Communications, 26(4):650-660, May 2008.

[6] C. Commault, J.-M. Dion, D.-H. Trinh, and T.-H. Do. Sensor classification for the fault detection and isolation, a structural approach Int. Journal of Adaptive Control and Signal Processing, 25:1-17, Jan. 2011.

[7] A. Rahmani, M. Ji, M. Mesbahi, and M. Egerstedt. Controllability of multi-agent systems from a graph-theoretic perspective. SIAM Journal on Control and Optimization, 48:162-186, 2009.

[8] M. Mesbahi and M. Egerstedt. Graph Theoretic Methods in Multiagent Networks. Princeton University Press, 2010.

[9] Y.Y. Liu, J.J. Slotine, and A.L. Barabasi. Controllability of complex networks. Nature, 473(7346):167-173, 2011.

[10] M. Ji and M. Egerstedt. Observability and estimation in distributed sensor networks. In Proc. IEEE Conf. Decision Control (CDC), pages 4221-4226, 2007.

[11] S. Zhang, K. Camlibel, and M. Cao. Controllability of diffusivelycoupled multi-agent systems with general and distance regular coupling topologies. In Proc. IEEE Conf. Decision Control (CDC), pages 759-764, 2011.

[12] M. Egerstedt, S. Martini, M. Cao, K. Camlibel, and A. Bicchi. Interacting with networks: How does structure relate to controllability in single-leader consensus networks? IEEE Control Systems Mag., 32(4):66-73, August 2012.

[13] G. Parlangeli and G. Notarstefano. On the reachability and observability of path and cycle graphs. IEEE Trans. on Automatic Control, 57(3):743-748, march 2012.

[14] G. Notarstefano and G. Parlangeli. Reachability and observability of simple grid and torus graphs. In Proc. IFAC World Congress, Milan, Italy, August 2011.

[15] G. Notarstefano and G. Parlangeli. Observability and reachability of grid graphs via reduction and symmetries. In Proc. IEEE CDC, Orlando, FL, USA, December 2011.

[16] A.E. Brouwer, A.M. Cohen, and A. Neumaier. Distance-regular graphs. Springer, 1989.

[17] Y. Zhou, Y. Fang, and Y. Zhang. Securing wireless sensor networks: a survey. IEEE Communications Surveys, 10(3):6-28, 2008.

[18] R.C. Bose and D.M. Mesner. On linear associative algebras corresponding to association schemes of partially balanced designs. The Annals of Mathematical Statistics, 30:21-38, 1959.

[19] R. Lopez-Valcarce and S. Dasgupta. On blind equalization of rank deficient nonlinear channels. In Proc. IEEE Workshop on Statistical Signal Proc. (SSP), Singapore, August 2001.

[20] C. Godsil and G. Royle. Algebraic graph theory. Springer, 2001. 